\title{
High frequency continuous birefringence-induced oscillations in spin-polarized vertical-cavity surface-emitting lasers
}

\author{
M. S. Torre ${ }^{1}$, H. Susanto ${ }^{2}$, Nianqiang LI ${ }^{3}$, K. SchiRes $^{4}$, M. F. Salvide ${ }^{1}$, I. D. Henning ${ }^{3}$, M. J. \\ AdAMS $^{3}$, AND A. HURTADO ${ }^{5, *}$ \\ ${ }^{1}$ Instituto de Fisica Arroyo Seco and CIFICEN (UNCPBA-CICPBA-CONICET), Tandil, Argentina \\ ${ }^{2}$ Department of Mathematics, University of Essex, Colchester, UK \\ ${ }^{3}$ School of Computer Science and Electronic Engineering, University of Essex, Colchester, UK \\ ${ }^{4}$ CNRS LTCI, Télécom ParisTech, Université Paris-Saclay, Paris, France \\ ${ }^{5}$ Institute of Photonics, SUPA Department of Physics, University of Strathclyde, Glasgow, UK \\ *Corresponding author: antonio.hurtado@strath.ac.uk

Sustained, large amplitude and tuneable birefringenceinduced oscillations are obtained in a spin-Vertical Cavity Surface Emitting Laser (spin-VCSEL). Experimental evidence is provided using a spin-VCSEL operating at $1300 \mathrm{~nm}$, under continuous wave optical pumping and at room temperature. Numerical and stability analyses are performed to interpret the experiments and to identify the combined effects of pump ellipticity, spin relaxation rate and cavity birefringence. Importantly, the frequency of the induced oscillations is determined by the device's birefringence rate which can be tuned to very large values. This opens the path for ultrafast spin-lasers operating at record frequencies exceeding those possible in traditional semiconductor lasers and with ample expected impact in disparate disciplines (e.g. datacomms, spectroscopy). ( 2017 Optical Society of America

OCIS codes: (140.0140) Lasers and laser optics; (140.5960) Semiconductor lasers; (140.7260) Vertical cavity surface emitting lasers.

http://dx.doi.org/10.1364/ol.XX.XXXXXX

The dynamics of vertical-cavity surface-emitting lasers (VCSELs) contain two dominant frequencies, namely (1) the relaxation oscillation frequency which is related to the geometric mean of the electron recombination rate and the photon decay rate, and (2) the frequency splitting between the two orthogonal linearly polarised modes which is related to cavity birefringence [1]. Experimental observations of oscillations of type (2) with a strong frequency component at $2.1 \mathrm{GHz}$ have been reported in $850 \mathrm{~nm}$ VCSELs [2,3]. More complex polarisation dynamics including polarisation switching, mode-hopping and chaos have also been observed [3-6]. Here, in addition to type (1) and (2) frequencies, bifurcation analysis (including continuation techniques [7]) serves to reveal richer behaviours. For spin-VCSELs where a spin-polarised electron population is achieved via either electrical injection using magnetic contacts or by optical pumping using circularly polarised light, the two dominant frequencies also determine the dynamics. Thus polarisation oscillations have been observed at $11.6 \mathrm{GHz}$ in a commercial VCSEL using a hybrid pumping scheme combining D.C. electrical with circularly-polarised optical pumping $[8,9]$. These are of type (2) and die away within 1 or 2 ns unless the device is operated close to the polarisation switching point (just above threshold). There the dichroism is minimised and the oscillations can last for about 5 ns [9]. Controlled switching of the oscillations using two optical pump pulses with variable delay has been demonstrated for a device with an oscillation frequency of $10.3 \mathrm{GHz}$ [10]. Since the frequency is controlled by the birefringence, the use of strain has been proposed as a way to tune the latter and thus attain higher frequencies [11]. By this means a birefringence splitting above $250 \mathrm{GHz}$ has been reported [12] and frequency tuning from $20 \mathrm{GHz}$ to $44 \mathrm{GHz}$ has been achieved [13]. Whilst the results above for a hybrid pumping scheme show damped oscillations, the present contribution deals with continuous undamped oscillations seen at frequency (2) using CW optical pumping (whose polarisation can be varied from linear through elliptical to circular, right or left). In previous works we have studied optically-pumped spin-VCSELs using dilute nitride materials and emitting at $1300 \mathrm{~nm}$ [14-17]. Experimental results on these devices have been simulated [14-16] using the spin-flip model (SFM) rate equations $[1,18,19]$ with a good level of agreement. We have also used SFM simulation to analyse the dynamics of spin-VCSELs $[15,20]$ and experimentally observed undamped polarisation oscillations tunable from $8.6 \mathrm{GHz}$ to $11 \mathrm{GHz}$ as the polarisation of the pump is varied [21]. The objective of the present contribution is to develop a fundamental understanding of this dynamic behaviour, highlighting the specific influence of a range of basic device properties and operating conditions. This is based on a combined approach using a recent theoreti- 


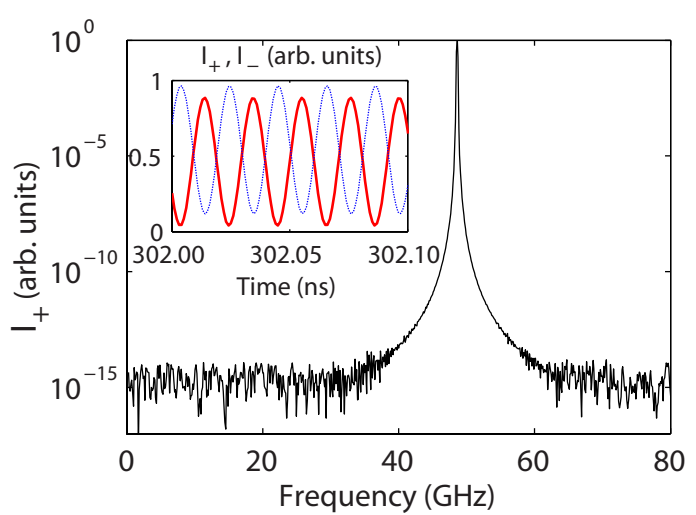

Fig. 1. Calculated normalized spectrum of $I_{+}$, with (inset) time dependence of circularly polarized output intensities: $I_{+}$ solid red line; $I_{-}$dashed blue line. The VCSEL parameters are: $\gamma_{p}=150 \mathrm{~ns}^{-1}, \gamma_{s}=63 \mathrm{~ns}^{-1}, \gamma=1 \mathrm{~ns}^{-1}, \gamma_{a}=0 \mathrm{~ns}^{-1}, \alpha=3$, $\kappa=250 \mathrm{~ns}^{-1}, \eta=2, P=-1$.

cal stability analysis [22] to provide insight into the nature and evolution of the dynamics, together with numerical simulations targeted on specific regions of interest informed by experimental observations. The stability analysis indicates that a Hopf bifurcation leads to these stable polarization oscillations and the agreement between numerical simulations and experimental findings is excellent. Moreover, the work reveals both routes for controlling these oscillations as well as prospects for very high frequency operation limited only by the birefringence rate. Since this can be made very high, up to hundreds of GHz [12], this offers in turn great prospects for novel, simple and inexpensive ultrafast laser sources with ample expected impact in data communications and spectroscopy applications.

Our theoretical analysis is based on the SFM coupled rate equations [1] in terms of right- and left-circularly polarized (RCP, LCP) field components, denoted by $E_{+}$and $E_{-}$, respectively, whose optical intensities are given by $I_{ \pm} \propto\left|E_{ \pm}\right|^{2}$. The spin relaxation rate is represented by $\gamma_{s}, \alpha$ is the linewidth enhancement factor, $\gamma$ is the electron density decay rate, $\kappa$ is the cavity decay rate and $\gamma_{p}$ and $\gamma_{a}$ correspond, respectively, to the linear birefringence and dichroism rates. The total optical pump rate applied to the VCSEL is $\eta=\eta_{+}+\eta_{-}$, where $\eta_{+}$and $\eta_{-}$are the RCP and LCP components. The ellipticity of the optical pump, $P$, is given by $P=\left(\eta_{+}-\eta_{-}\right) / \eta$ and that of the output of the spin-VCSEL is defined as $\varepsilon=\left(I_{+}-I_{-}\right) /\left(I_{+}+I_{-}\right)$. It should be noted that we assume that in the case of quantum well active media where the degeneracy of heavy hole $(h h)$ and light hole $(l h)$ states is lifted, it is a reasonable approximation to ignore transitions between the conduction band and the $l h$ states.

As an example of the oscillations under discussion, Fig. 1 shows the calculated radiofrequency (RF) spectrum of $I_{+}$for LCP pumping $(P=-1)$, with (inset) simulated time series for the spin-VCSEL's RCP and LCP intensities, $I_{+}$and $I_{-}$. The VCSEL parameters are indicated in the caption of fig. 1 . For this set of parameter values the relaxation oscillation frequency of the device was equal to $\approx 3.6 \mathrm{GHz}$. Fig. 1 shows that the two circularly polarized components exhibit sustained, large-amplitude periodic oscillations at a frequency given by the birefringence frequency $\gamma_{p} / \pi(=47.75 \mathrm{GHz}$ in this case). It was also found that the value of the spin relaxation rate $\gamma_{s}$ has a negligible effect on the frequency of the oscillations, but smaller values of $\gamma_{s}$ were benefitial for oscillations to occur at higher values of birefringence and therefore at higher frequencies [20].

Numerical solution of the SFM equations (as in Fig. 1) provides time-dependent results for specific sets of parameters, but does not give a complete description of stable and unstable solutions, their nature and boundaries, and the trends in their behaviour with variation of model parameters. Thus we carried out a complementary stability analysis which enables us to explain the basis of the oscillatory phenomena of Fig. 1. The method, described in [22], is founded on perturbation of the steady-state solutions for general elliptically-polarized fields, which are characterised by a constant phase difference between the RCP and LCP components. By analogy with the case of linear polarization where this phase difference is either 0 or $\pi$, the solutions are termed in-phase and out-of-phase, corresponding to cases where this phase difference is a continuation of either 0 or $\pi$, respectively. The perturbation technique leads to an eigenvalue problem whose solution determines the underlying dynamics and allows us to explore the trends with model parameters. The real part of the critical eigenvalue determines the stability of the steady-state solutions; the solution is unstable when there is a complex-valued eigenvalue $\lambda$ with $\operatorname{Re}(\lambda)>0$ and stable provided that $\operatorname{Re}(\lambda)<0$ for all eigenvalues. For the problem here we show that a pair of eigenvalues (i.e., critical eigenvalues) characterises the instability. To understand the mechanisms leading to the oscillatory behaviour, the evolution of $\operatorname{Re}(\lambda)$ for the in-phase and out-of-phase solutions was studied as a function of model parameters.

In Fig. 2(a) we present results for $\operatorname{Re}(\lambda)$ as a function of the spin relaxation rate $\gamma_{s}$, with the rest of parameters as in Fig. 1. Three distinct regions of operation can be identified; two regions where out-of-phase (low values of $\gamma_{s}$ ) and in-phase (high values of $\gamma_{s}$ ) solutions are stable, and an oscillatory region (the highlighted yellow region), where only time-periodic solutions are allowed as there is no stable equilibrium. In this region, the underlying attractor changes from a fixed point to a limit cycle at the supercritical Hopf bifurcation (HB) point (at $\gamma_{s}=62 \mathrm{~ns}^{-1}$ ), yielding a large amplitude periodic oscillation with the frequency (determined by the imaginary part of the critical eigenvalue at the bifurcation point) being close to the birefringence frequency. An example of the time trace corresponding to this behaviour is depicted in the inset of Fig. 1. In the (yellow) oscillatory region, the system remains in a stable oscillatory state, i.e., in the region of attraction of the limit cycle, until it reaches an inverse $\mathrm{HB}$ at $\gamma_{s}=67 \mathrm{~ns}^{-1}$ where the system
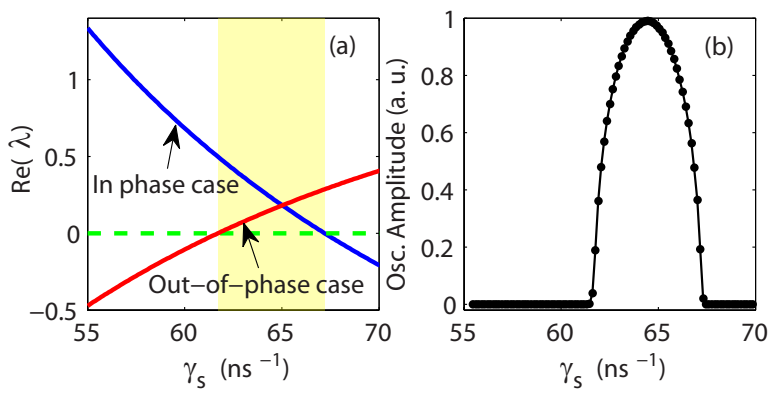

Fig. 2. (a) Real part of the critical eigenvalue $\lambda$ as a function of $\gamma_{s}$ for in-phase and out-of-phase solutions (b) Calculated oscillation amplitude of $I_{+}$as a function of $\gamma_{s}$. VCSEL parameters are the same as in Fig. 1. 


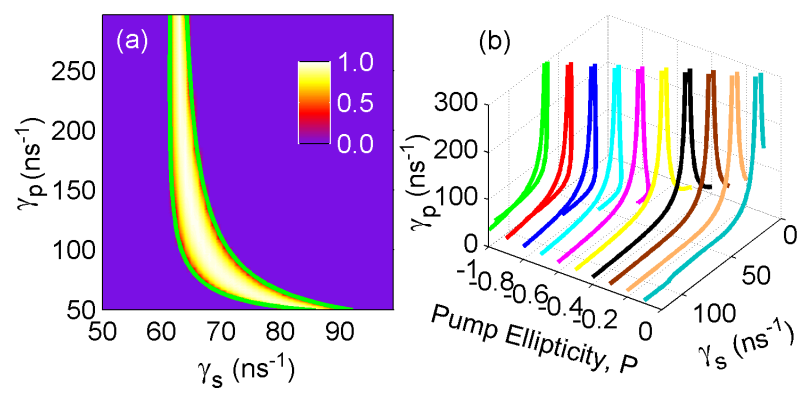

Fig. 3. (a) Calculated contour map in the $\gamma_{s}-\gamma_{p}$ plane for the average oscillation amplitude of $I_{+}$with superimposed stability boundaries (green). (b) Variation of stability boundaries in the $\gamma_{s}-\gamma_{p}$ plane with pump ellipticity $P$. The other VCSEL parameters are as in Fig. 1.

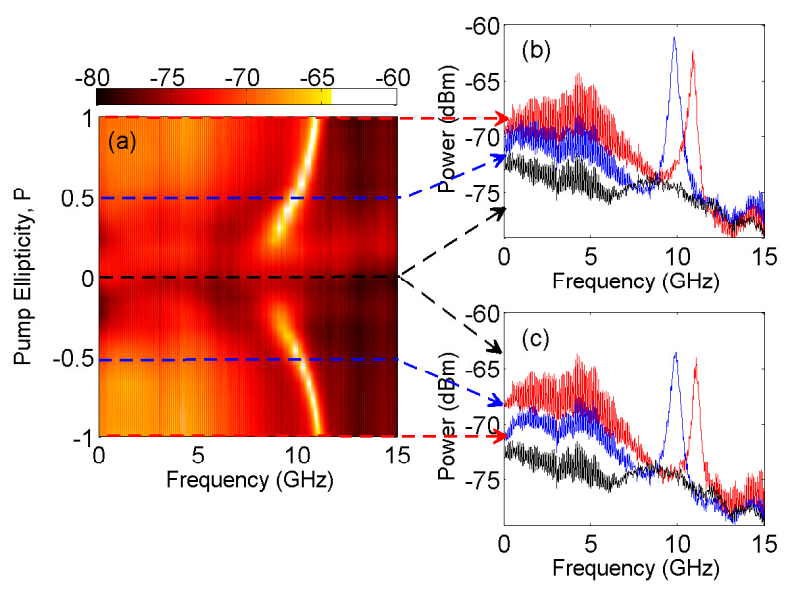

Fig. 4. Measured evolution of the spin-VCSEL's RF spectrum with pump polarization. Insets show the RF spectra at $P=$ $\pm 1, \pm 0.5$, and 0 .

returns to stable in-phase solutions. Fig. 2(b) shows the variation of oscillation amplitude vs. $\gamma_{s}$ obtained solving numerically the SFM equations. As expected, the window for oscillatory behavior obtained in Fig. 2(b) is identical to that in Fig. 2(a).

Fig. 3(a) shows a numerically simulated map in the $\gamma_{s}-\gamma_{p}$ plane with colour contours of the amplitude of oscillations found in the RCP component of intensity, $I_{+}$(all other model parameters as in Fig. 1). This confirms a window of $\gamma_{s}$ values where strong oscillatory behaviour is obtained even as $\gamma_{p}$ approaches very high values $\sim 300 \mathrm{~ns}^{-1}$ (over $95 \mathrm{GHz}$ in frequency). The solid green lines, calculated now using the new stability analysis, plot the HB boundaries where strong oscillations occur, showing excellent agreement with the numerical results. In Fig. 3(b) we use the stability analysis to plot the evolution with pump ellipticity $(P)$ of the stability boundaries in the $\gamma_{s}-\gamma_{p}$ plane (all other parameters as in Fig. 1). Variation of $P$ shifts the boundaries towards higher values of $\gamma_{s}$ as the pump polarization changes from linear $(P=0)$ to LCP $(P=-1)$. This demonstrates the potential to use $P$ to tune the system, thus opening the door to externally control of the oscillatory state of the spin-VCSEL.

We now focus on the experimental study of periodic oscillations in a spin-VCSEL. For complete details on the VCSEL-wafer including diagrams of its structure and photoluminescence and reflectivity spectral measurements see [14]. The top and bottom

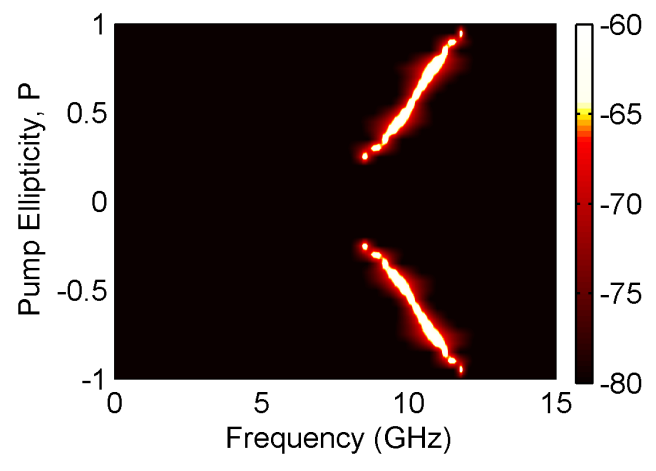

Fig. 5. Simulated evolution of the spin-VCSEL's RF spectrum with pump polarization. Parameter values are: $\gamma_{s}=110 \mathrm{~ns}^{-1}$, $\gamma_{p}=27.64 \mathrm{~ns}^{-1}, \gamma=0.68 \mathrm{~ns}^{-1}, \gamma_{a}=0 \mathrm{~ns}^{-1}, \kappa=230 \mathrm{~ns}^{-1}$, $\alpha=4, \eta=5$.
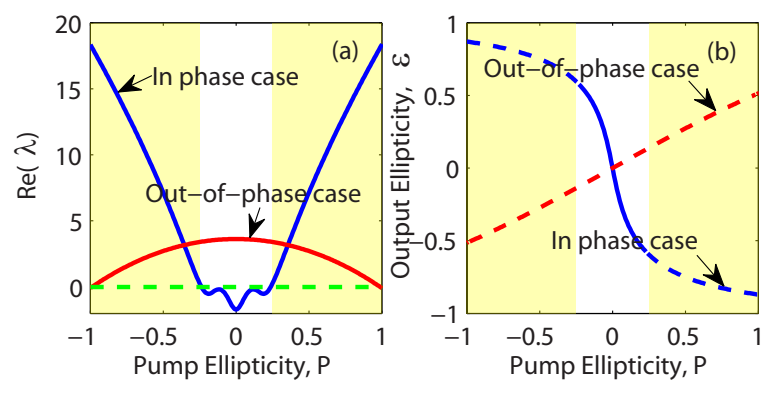

Fig. 6. (a) Real part of the critical eigenvalue $\lambda$ and (b) output ellipticity $\varepsilon$ as a function of the pump ellipticity $P$ for inphase and out-of-phase solutions. In (b) unstable and stable solutions are indicated as broken and solid lines, respectively. VCSEL parameters are as in Fig. 5.

Bragg mirrors of the VCSEL-wafers were designed with 16 and $20.5 \mathrm{GaAs} / \mathrm{AlAs}$ pairs, respectively, providing high reflectivities of 0.992 and 0.998 . These mirrors enclosed a $3-\lambda$ cavity with five groups of three $7 \mathrm{~nm} G a_{0.67} I n_{0.33} N_{0.016} A s_{0.984}$ quantum wells sandwiched between $2 \mathrm{~nm} \mathrm{Ga} a_{0.75} \operatorname{In}_{0.25} N_{0.017} A s_{0.983}$ strain mediating layers and located approximately at the antinodes of the optical field. The VCSEL wafer was mounted on a copper stage and its temperature was kept constant at $293 \mathrm{~K}$ throughout the experiments. Polarization-controlled continuouswave optical pumping with a $980 \mathrm{~nm}$ pump laser was used to excite spin lasing at $1300 \mathrm{~nm}$ in a VCSEL wafer at room temperature. The wafer was optically pumped through a lens-ended fibre giving a spot diameter of approximately $10 \mu \mathrm{m}$. The pump polarization was adjusted with an in-line polarization controller, so that the pump ellipticity was chosen as the control parameter. More details on the experimental study of the spin-VCSEL polarization-resolved dynamics can be found in [14, 21]. Fig. 4(a) shows a high-resolution mapping of the measured RF spectrum at the spin-VCSEL's output with varying pump polarization, $P$, from -1 (LCP) to 1 (RCP) polarization. In Fig. 4(a) the colour indicates measured RF power $(\mathrm{dBm})$ with brighter (darker) hues for higher (lower) measured RF power. Figs. 4(b) and 4(c) plot measured RF spectra for values of pump ellipticity: $P= \pm 1, \pm 0.5$, and 0 , showing peaks corresponding to sustained oscillations whose frequency increases as $|P|$ is increased from $\approx 0.25$ to 1 , while no peaks are seen for $|P| \leq 0.25$ (note that the system is 
symmetric about $P=0)$. As predicted in Fig. 3(b), the experiments confirm that when $|P|$ exceeds a critical value the system crosses a stability boundary, transiting from a stable region to one of oscillatory behaviour. Here, the oscillation frequency can be tuned from $\sim 8.6 \mathrm{GHz}$ to $\sim 11 \mathrm{GHz}$ as $P$ is increased from 0.25 to 1 . This behaviour differs from the damped oscillations reported previously in [8-10,13] which fade away within a few ns. We must point out here that no time traces measurements of the oscillations were possible as our equipment was not sufficiently fast to resolve them. However, the device was operated under continuous wave optical pumping and the spectral analyses revealed that RF results could be obtained reproducibly, thus indicating the presence of continuous oscillations at the spin-VCSEL's output.

Fig. 5 shows the corresponding simulated results using the SFM. Here the specific set of VCSEL parameters were chosen for best agreement with the experiment results, and these are given in the caption. For those parameter values the relaxation oscillation frequency of the device was equal to $\approx 5.6 \mathrm{GHz}$. We should mention that the measured optical spectra of our spinVCSEL did not have enough resolution to derive empirically the birefringence rate value and its value was set equal to $\gamma_{p}=27.64$ $\mathrm{ns}^{-1}$ to obtain the best fit with the experimental findings. For direct comparison, the calculated RF amplitude is plotted with the same colour-coded method used for the experimental plot. The theoretical and experimental RF spectra show very good agreement in terms of oscillation frequencies and the range of $P$ for oscillatory behaviour. Additionally, good agreement between theory and experiments was also obtained when analyzing the evolution of the RF power as a function of $P$. We now apply the linear stability analysis to examine the in-phase and out-of-phase solutions as the pump ellipticity $P$ is varied from -1 to 1 . Fig. 6 shows the evolution of the real part of the critical eigenvalue (Fig. 6(a)) and output ellipticity, $\varepsilon$ (Fig. 6(b)) as functions of $P$ for the same parameter values used in Fig. 5. The two highlighted yellow regions indicate where there is no stable equilibrium and therefore the system yields periodic solutions. Outside these regions the system exhibits stable in-phase solutions with no oscillations. The HB occurring at $|P| \approx 0.25$ is supercritical, marking the transition to a stable periodic solution at a frequency close to the birefringence frequency. These oscillations and their ranges correspond to those observed experimentally in Fig. 4 and simulated by numerical integration in Fig. 5 .

In summary, we report theoretically and experimentally on birefringence-induced continuous oscillations in a spin-VCSEL. Stability analysis reveals Hopf bifurcations which bound regions of limit cycles yielding sustained, large-amplitude oscillations at a frequency determined by the birefringence rate. Numerical simulations confirm these overall findings with excellent agreement. Importantly, means of external control (by the pump polarization) of these oscillations and their frequency is predicted theoretically and confirmed experimentally. These results offer the prospect of an engineering path for simple inexpensive ultrafast spin-laser sources with expected direct modulation bandwidths only limited by the birefringence rate $(\sim$ hundreds of $\mathrm{GHz}$ ), thus overcoming the limitations imposed by the relaxation oscillation frequency in traditional semiconductor lasers and with high potential for applications as ultrafast sources in high-speed optical communication and spectroscopy systems.

The work was supported by the UK Engineering and Physical Sciences Research Council (grants EP/M024237/1 and EP/G012458/1) and the University of Strathclyde through the
Chancellor's Fellowship Program. The authors are grateful to V.M. Korpijarvi and M. Guina (Tampere University of Technology, Finland) for supply of the VCSEL wafer.

\section{REFERENCES}

1. A. Gahl, S. Balle, and M. S. Miguel, IEEE J. Quantum Electron. 35, 342 (1999)

2. T. Ackemann and M. Sondermann, Appl. Phys. Lett. 78, 3574 (2001).

3. M. Sondermann, T. Ackemann, S. Balle, J. Mulet, and K. Panajotov, Opt. Commun. 235, 421 (2004).

4. L. Olejniczak, K. Panajotov, H. Thienpont, M. Sciamanna, A. Mutig, F. Hopfer, and D. Bimberg, Opt. Express 19, 2476 (2011).

5. M. Virte, K. Panajotov, H. Thienpont, and M. Sciamanna, Nat. Photonics 7, 60 (2013).

6. M. Marconi, J. Javaloyes, S. Barland, S. Balle, and M. Giudici, Nat. Photonics 9, 450 (2015).

7. M. Virte, K. Panajotov, and M. Sciamanna, Phys. Rev. A 87, 013834 (2013).

8. M. Li, H. Jähme, H. Soldat, N. Gerhardt, M. Hofmann, and T. Ackemann, Appl. Phys. Lett. 97, 191114 (2010).

9. N. Gerhardt, M. Li, H. Jähme, H. Höpfner, T. Ackemann, and M. Hofmann, Appl. Phys. Lett. 99, 151107 (2011).

10. H. Höpfner, M. Lindemann, N. C. Gerhardt, and M. R. Hofmann, Appl. Phys. Lett. 104, 022409 (2014).

11. P. E. F. Junior, G. Xu, J. Lee, N. C. Gerhardt, G. M. Sipahi, and I. Žutić, Phys. Rev. B 92, 075311 (2015).

12. T. Pusch, M. Lindemann, N. C. Gerhardt, M. R. Hofmann, and R. Michalzik, Electron. Lett. 51, 1600 (2015).

13. M. Lindemann, T. Pusch, R. Michalzik, N. C. Gerhardt, and M. R. Hofmann, Appl. Phys. Lett. 108, 042404 (2016).

14. K. Schires, R. Al Seyab, A. Hurtado, V.-M. Korpijärvi, M. Guina, I. D. Henning, and M. J. Adams, Opt. Express 20, 3550 (2012).

15. S. S. Alharthi, R. Al Seyab, I. D. Henning, and M. J. Adams, IET Optoelectron. 8, 117 (2014)

16. S. S. Alharthi, A. Hurtado, R. Al Seyab, V.-M. Korpijarvi, M. Guina, I. D. Henning, and M. J. Adams, Appl. Phys. Lett. 105, 181106 (2014).

17. S. S. Alharthi, A. Hurtado, V.-M. Korpijarvi, M. Guina, I. D. Henning, and M. Adams, Appl. Phys. Lett. 106, 021117 (2015).

18. M. San Miguel, Q. Feng, and J. Moloney, Phys. Rev. A 52, 1728 (1995).

19. J. Martin-Regalado, F. Prati, M. S. Miguel, and N. Abraham, IEEE J. Quantum Electron. 33, 765 (1997).

20. R. Al Seyab, D. Alexandropoulos, I. D. Henning, and M. J. Adams, IEEE Photon. J. 3, 799 (2011).

21. K. Schires, R. Al Seyab, A. Hurtado, V.-M. Korpijärvi, M. Guina, I. D. Henning, and M. J. Adams, in Photonics Conference (IPC), 2012 IEEE (IEEE, 2012) pp. 870-871.

22. H. Susanto, K. Schires, M. J. Adams, and I. D. Henning, Phys. Rev. A 92, 063838 (2015). 


\section{REFERENCES}

1. A. Gahl, S. Balle, and M. S. Miguel, "Polarization dynamics of optically pumped VCSELs," IEEE J. Quantum Electron. 35, 342 (1999).

2. T. Ackemann and M. Sondermann, "Characteristics of polarization switching from the low to the high frequency mode in vertical-cavity surface-emitting lasers," Appl. Phys. Lett. 78, 3574 (2001).

3. M. Sondermann, T. Ackemann, S. Balle, J. Mulet, and K. Panajotov, "Experimental and theoretical investigations on elliptically polarized dynamical transition states in the polarization switching of verticalcavity surface-emitting lasers," Opt. Commun. 235, 421 (2004).

4. L. Olejniczak, K. Panajotov, H. Thienpont, M. Sciamanna, A. Mutig, F. Hopfer, and D. Bimberg, "Polarization switching and polarization mode hopping in quantum dot vertical-cavity surface-emitting lasers," Opt. Express 19, 2476 (2011).

5. M. Virte, K. Panajotov, H. Thienpont, and M. Sciamanna, "Deterministic polarization chaos from a laser diode," Nat. Photonics 7, 60 (2013).

6. M. Marconi, J. Javaloyes, S. Barland, S. Balle, and M. Giudici, Nat. Photonics 9, 450 (2015).

7. M. Virte, K. Panajotov, and M. Sciamanna, "Bifurcation to nonlinear polarization dynamics and chaos in vertical-cavity surface-emitting lasers," Phys. Rev. A 87, 013834 (2013).

8. M. Li, H. Jähme, H. Soldat, N. Gerhardt, M. Hofmann, and T. Ackemann, "Birefringence controlled room-temperature picosecond spin dynamics close to the threshold of vertical-cavity surface-emitting laser devices," Appl. Phys. Lett. 97, 191114 (2010).

9. N. Gerhardt, M. Li, H. Jähme, H. Höpfner, T. Ackemann, and M. Hofmann, "Ultrafast spin-induced polarization oscillations with tunable lifetime in vertical-cavity surface-emitting lasers," Appl. Phys. Lett. 99, 151107 (2011).

10. H. Höpfner, M. Lindemann, N. C. Gerhardt, and M. R. Hofmann, "Controlled switching of ultrafast circular polarization oscillations in spinpolarized vertical-cavity surface-emitting lasers," Appl. Phys. Lett. 104, 022409 (2014).

11. P. E. F. Junior, G. Xu, J. Lee, N. C. Gerhardt, G. M. Sipahi, and I. Žutić, "Toward high-frequency operation of spin lasers," Phys. Rev. B 92, 075311 (2015).

12. T. Pusch, M. Lindemann, N. C. Gerhardt, M. R. Hofmann, and R. Michalzik, "Vertical-cavity surface-emitting lasers with birefringence splitting above $250 \mathrm{GHz}$," Electron. Lett. 51, 1600 (2015).

13. M. Lindemann, T. Pusch, R. Michalzik, N. C. Gerhardt, and M. R. Hofmann, "Frequency tuning of polarization oscillations: Toward highspeed spin-lasers," Appl. Phys. Lett. 108, 042404 (2016).

14. K. Schires, R. Al Seyab, A. Hurtado, V.-M. Korpijärvi, M. Guina, I. D. Henning, and M. J. Adams, "Optically-pumped dilute nitride spinVCSEL," Opt. Express 20, 3550 (2012).

15. S. S. Alharthi, R. Al Seyab, I. D. Henning, and M. J. Adams, "Simulated dynamics of optically pumped dilute nitride $1300 \mathrm{~nm}$ spin vertical-cavity surface-emitting lasers," IET Optoelectron. 8, 117 (2014).

16. S. S. Alharthi, A. Hurtado, R. Al Seyab, V.-M. Korpijarvi, M. Guina, I. D. Henning, and M. J. Adams, "Control of emitted light polarization in a $1310 \mathrm{~nm}$ dilute nitride spin-vertical cavity surface emitting laser subject to circularly polarized optical injection," Appl. Phys. Lett. 105, 181106 (2014).

17. S. S. Alharthi, A. Hurtado, V.-M. Korpijarvi, M. Guina, I. D. Henning, and M. Adams, "Circular polarization switching and bistability in an optically injected $1300 \mathrm{~nm}$ spin-vertical cavity surface emitting laser," Appl. Phys. Lett. 106, 021117 (2015).

18. M. San Miguel, Q. Feng, and J. Moloney, "Light-polarization dynamics in surface-emitting semiconductor lasers," Phys. Rev. A 52, 1728 (1995).

19. J. Martin-Regalado, F. Prati, M. S. Miguel, and N. Abraham, "Polarization properties of vertical-cavity surface-emitting lasers," IEEE J. Quantum Electron. 33, 765 (1997).

20. R. Al Seyab, D. Alexandropoulos, I. D. Henning, and M. J. Adams, "Instabilities in spin-polarized vertical-cavity surface-emitting lasers," IEEE Photon. J. 3, 799 (2011).

21. K. Schires, R. Al Seyab, A. Hurtado, V.-M. Korpijärvi, M. Guina, I. D. Henning, and M. J. Adams, "Instabilities in optically-pumped 1300nm dilute nitride spin-VCSELs: experiment and theory," in Photonics Conference (IPC), 2012 IEEE (IEEE, 2012) pp. 870-871.

22. H. Susanto, K. Schires, M. J. Adams, and I. D. Henning, "Spin-flip model of spin-polarized vertical-cavity surface-emitting lasers: Asymptotic analysis, numerics, and experiments," Phys. Rev. A 92, 063838 (2015). 